Rabaska

Revue d'ethnologie de l'Amérique française

\title{
Littérature orale ou folklore : les frontières discursives et la " nuance qui fiance "
}

\section{Maurice Lamothe}

Volume 3, 2005

URI : https://id.erudit.org/iderudit/201716ar

DOI : https://doi.org/10.7202/201716ar

Aller au sommaire du numéro

Éditeur(s)

Société québécoise d'ethnologie

ISSN

1703-7433 (imprimé)

1916-7350 (numérique)

Découvrir la revue

Citer cette note

Lamothe, M. (2005). Littérature orale ou folklore : les frontières discursives et la « nuance qui fiance ». Rabaska, 3, 117-123. https://doi.org/10.7202/201716ar d'utilisation que vous pouvez consulter en ligne.

https://apropos.erudit.org/fr/usagers/politique-dutilisation/ 


\title{
Opinion
}

\section{Littérature orale ou folklore : les frontières discursives et la « nuance qui fiance »}

\author{
Maurice Lamothe \\ Université Saine-Anne, Pointe-de-l'Église
}

Quand on m'a demandé de participer à un comité dont le mandat serait de monter un cours universitaire sur les littératures orales, je me suis demandé ce qu'un littéraire pouvait bien apporter à des ethnologues et folkloristes. La tendance à la multidisciplinarité, ou à celle de la transversalité dont sont friands les pédagogues ces jours-ci, ne peut certes pas tout expliquer. Peutêtre est-ce tout simplement méprise ? Après tout, on confond souvent ma spécialité, la chanson populaire, avec la chanson folklorique. Mais ce serait là accorder trop peu de jugement à mes collègues.

On pourrait penser que le domaine de la littérature que je représente serait susceptible d'apposer une sorte de sceau à une tradition orale (contes, légendes et chansons) dont on ne reconnaît encore que bien timidement qu'elle peut recouvrer un caractère littéraire. Remarquons ici au passage que l'adoption d'un cours de littérature orale dans un département de littératures ne passe jamais inaperçu.

En fait, la meilleure raison de s'intéresser à un littéraire n'est peut-être pas celle que m'ont avouée mes collègues ethnologues et folkloristes. Elle tiendrait au fait que, malgré certaines réticences, il arrive que les départements de littératures voient à travers la littérature orale un outil de sensibilisation à la littérature écrite, celle que l'on ne veut pas lire et qui, pourtant, permettrait d'augmenter les compétences linguistiques des étudiants, pains et beurre de nos départements, étudiants qui du reste ne sont guère légion dans les départements de folklore ou d'ethnologie, lorsqu'il y en a un... De là à penser que mes collègues s'intéressent à moi pour les étudiants que je pourrais leur apporter, il n'y qu'un pas...

Peut-être en fait découvre-t-on qu'il n'y a pas de petite littérature, mais bien plutôt que de petits littéraires. Le terme de « paralittérature » serait donc ici à proscrire dans la mesure où il n'y aurait que « la littérature ». Notons en 
contrepartie l'existence de petits folkloristes dressant artificiellement des frontières sémantiques au nom d'une pureté qui laisse songeur. Nous voilà donc au cœur de notre sujet. De part et d'autre, la petitesse contribuerait à morceler le champ littéraire, laissant alors croire à l'achèvement de l'un puis à l'apparition de l'autre. Le défi consisterait donc ici à montrer qu'il y a interpénétration, c'est-à-dire inachèvement; ce qui aurait dû paraître évident pour des folkloristes entraînés pourtant à colliger les innombrables versions de toutes sortes dont l'ensemble pourrait être représenté par un rhizome inextricable, sans début, ni fin, ni centre : pensons ici, entres autres, aux 356 versions d'A la claire fontaine.

À ce morcellement du champ littéraire, il faudrait donc opposer une transgression à laquelle participerait un élan multidisciplinaire, terme aux connotations bien peu poétiques, mais qu'un Verlaine n'aurait sans doute pas renié sur le principe dans Art poétique : « la nuance seule fiance ${ }^{1}$ ». Le poète ajoute : « prends l'éloquence et tors-lui son cou ». C'est que la transgression attise les tensions de frontières, cette éloquence discursive donc qui se dresse comme autant de moulins dans le brouillard.

De sorte que, loin de rejeter l'invitation de mes collègues d'autres disciplines, j'aimerais plutôt apporter ici, en guise de dot, cette « nuance qui fiance » que $j$ 'opposerais aux frontières discursives que dressent de multiples objections ou préjugés souvent entretenus au nom d'une intégrité territoriale, une attitude de défiance qu'alimente un discours sur l'identité et la modernité.

\section{Première affirmation: Dès qu'il y a un auteur, il n'y a plus de folklore.}

Pour Conrad Laforte, Partons la mer est belle appartient à la catégorie des chansons littéraires, une fausse catégorie, dit-il. Bien qu'on ne connaisse pas l'auteur de la chanson bretonne, celle-ci serait en fait trop bien construite pour être considérée comme folklorique. Il y aurait donc ici corrélation entre la qualité esthétique et l'existence d'un auteur, lesquelles seraient consubstantielles à un caractère folklorique douteux.

Le paradigme « droit d'auteur » paraît dès lors intéressant. En Louisiane pourtant, il pose problème. Au début du siècle dernier, le couple Falcon enregistre Allons à Lafayette, une des plus vieilles chansons cajuns attestées dont le caractère folklorique ne peut être mis en doute. La chanson rejoint en fait toutes les autres Allons danser Colinda et Jambalaya du musée de Vermillionville près de Lafayette. Enfin, " Amérique » oblige, leurs auteurs sont souvent des vedettes. Il arrive aussi qu'ils soient toujours vivants tandis que les débats sur les droits d'auteurs font encore des ravages. Tiguilaguilo me dit un jour un des frères Clément de la région Évangéline, « c'est moi qui

1. Verlaine, CEuvres poétiques, (Art poétique), Paris, Bordas, 1985, p. 99. 
l'ai composée mais je n'ai eu que cinquante piastres pour ça. Y en a un qui a fait une fortune avec ça et qui fait croire que c'est lui l'a composée ».

En Louisiane, si le conflit entre identité et modernité ne semble guère forcer une distinction entre chanson folklorique et chanson à auteur, en revanche, on se surprendra de l'absence d'un certain Zachary Richard des murs du musée de Vermillionville. À moins cependant que l'on sache que Barry Ancelet, ethnologue de Lafayette et responsable de la mise en forme du musée, a écrit que Zachary Richard représentait davantage les attentes du marché extérieur que celles de la Louisiane. Nos études à ce sujet montrent en fait que le parcours du célèbre auteur de Réveille, se caractérise par un éloignement de la chanson à danser et un rapprochement clair vers la chanson à texte, pour tout dire : l'écrit.

\section{Deuxième affirmation : Le folklore est incompatible avec la modernité.}

Il s'agit là d'une accusation souvent notée aussi en chanson populaire contemporaine. Cette peur de ne pas être de son temps fait des ravages. Le cas de la Bolduc est particulièrement intéressant ici. Même si elle a été boudée par la radio naissante de Montréal pour qui la modernité ne pouvait être qu'exogène (cha-cha-cha, rumbas et autres rythmes latins), on ne pouvait empêcher que la lutte rime avec la turlutte et que Montréal fasse rhizome jusqu'à Chéticamp et à la Baie Sainte-Marie. En 1936 en effet, trois malades de l'hôpital de Chéticamp, pour mieux se plaindre de leurs conditions, réinventent les paroles d'une chanson de la Bolduc : « Ça va venir, ça va

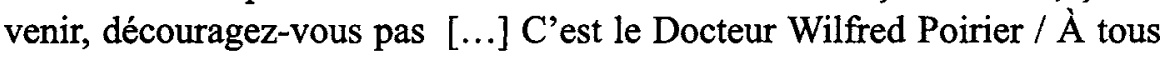
les jours il vient nous visiter / Quand il viendra ôter nos points / Ce sera plus encourageant $»$. De sorte que même si, main dans la main, la chanson d'auteur et les médias contribuent à fixer le produit dans le temps, les dérives demeurent fréquentes, volontaires même, permettant à l'occasion d'espérer une emprise accrue sur le réel.

Il faut dire ici que cette trouvaille sur le terrain fut pour moi une authentique révélation que je dois à la patience de la démarche archivistique que m'ont inspirée mes collègues. Bien que l'on se doutât bien que l'avantBolduc et l'après-Bolduc ne correspondaient pas obligatoirement à une frontière claire entre le folklore et la chanson contemporaine, l'exemple de cette chanson L'Hôpital de Chéticamp ${ }^{2}$ brouille encore davantage les pistes en présentant, au surplus, un comportement " versionniste ", typique de la chanson folklorique (les chansons à timbres par exemple), vis-à-vis d'un produit qui n'a pu se rendre aussi loin et aussi rapidement que grâce à des

2. Collection Léo Aucoin, Centre acadien de l'Université Sainte-Anne, Pointe-de-l'Église, NouvelleÉcosse. 
moyens techniques modernes comme la radio ou le disque que l'auteur aura peut-être distribué lors d'une tournée en Gaspésie.

La première vague de chansonniers francophones hors Québec des années soixante-dix et ce qu'il en advint.

Dès 1980, la chanson francophone hors Québec dans son ensemble a été particulièrement éprouvée par une étiquette folklorique que lui accolait la critique. Trop folklorique, ou pas assez... Trop moderne ou pas assez... La chanson francophone hors Québec entre alors dans une crise profonde qui allait l'obliger à se transformer et, dans certains cas, à revenir à la maison. Ainsi, s'il pouvait sembler paradoxal que, au nom de "leur spécificité », les chansonniers hors Québec aient participé à l'élan nationaliste québécois des années soixante-dix alors que l'Association des francophones hors Québec a toujours vu la séparation du Québec comme un mouvement d'abandon des spécificités, c'est que l'on oublie à quel point le marché québécois est vital pour un produit culturel francophone hors Québec, au point que les contradictions deviennent bien secondaires lorsque comparées aux bénéfices que peut apporter une mise en marché efficace.

L'étiquette " folklore " agit tout à coup comme un boulet au pied de la chanson francophone contemporaine hors Québec.

Alors qu'en 1987, Pierre Boivin, de Kébec-Disc, accusait les chansonniers francophones hors Québec de contribuer à leur perte en continuant à « chanter leur patrimoine » (comme à l'époque des «folkeux » des années soixantedix), Paul Tanguay cherche à démarquer son produit de toute espèce de relation avec l'étiquette « folklore ». Il va même jusqu'à établir une distinction entre « musique folklorique » et " musique traditionnelle ». Lorsqu'il est question des chansonniers franco-ontariens comme Donald Poliquin et Michel Lalonde, Tanguay éprouve le besoin d'affirmer avec vigueur ce que le produit francoontarien n'est pas en 1989 :

[...] non, ce n'est pas du folklore, c'est de la musique traditionnelle, un peu comme une musique classique. Ce n'est pas du tout du folklore, les gars ne se promènent pas avec des ceintures fléchées [...]. Ce n'est pas de la musique folklorique, c'est de la musique traditionnelle... On ne charrie pas d'image de folklore, il n'y a pas de sirop d'érable sur la scène, de ceintures fléchées [...] en chemise « carreautée » avec des cuillères ${ }^{3}$.

Par ailleurs, Tanguay prétend que Garolou (le groupe dont Michel Lalonde était le leader) n'a jamais fait de folklore. Selon lui, il s'agissait plutôt de

3. Entrevue avec Paul Tanguay, accordée à Maurice Lamothe, Montréal, 28 octobre 1989, 16 p. 
rock $^{4}$. S'il n'est pas question ici d'engager un débat sur les genres musicaux, on peut en revanche se demander si l'insistance de Tanguay à prétendre qu'il ne vend pas de folklore ne trahit pas les stigmates d'une défensive bien rodée contre un préjugé " folkeux » entretenu envers les chansonniers francocanadiens et acadiens pendant la décennie des années quatre-vingt.

Ici, il faut éviter de parler de simple malentendu. Il est question plutôt d'identifier la nature des forces qui sont en jeu au sein de l'institution de la chanson francophone au Québec et au Canada, et espérer ensuite faire la lumière sur la nécessité d'un certain discours qui contribue à établir une tension entre le champ québécois de la chanson et celui de la chanson francoontarienne. Ainsi, entrent en opposition le discours de Boivin sur les chansonniers francophones hors Québec et celui de Tanguay, qui clame que la chanson franco-ontarienne n'est plus folklorique. En somme, il ne s'agit donc pas de déterminer si, durant les années quatre-vingt, l'étiquette folklorique est réellement aussi populaire auprès des chansonniers francophones hors Québec que Boivin le prétend, mais de voir dans quelle mesure ce « folklorisme » dont Boivin accuse les chansonniers hors Québec - et auquel Tanguay refuse de souscrire - n'est pas le lieu d'une bataille rangée pour élargir un marché d'un côté comme de l'autre ${ }^{5}$.

Ce rejet de l'étiquette folklorique chez Boivin et Tanguay durant les années quatre-vingt procède d'un seul et même souci : celui, en l'occurrence, d'un producteur de disques et d'un gérant-producteur de spectacles qui cherchent à réagir face à un marché dont les conditions ont changé. Dans la mesure où le folklore est associé à l'idéologie nationaliste des années soixantedix, il devient impérieux de proposer un produit dont l'image est renouvelée.

En somme ici, la «non-modernité » du produit chansonnier tient davantage d'un changement dans les attentes du public qu'à une caractéristique intrinsèque au produit.

\section{Troisième affirmation : Faire du folklore équivaut à donner dans l'" ethnic business ${ }^{6} \%$}

Il arrive en effet que ce soit le cas, que le discours critique allie folklore et recherche de profits à court terme. C'est d'ailleurs un peu ce que prétend affirmer l'expression « folkeux » évoquée plus haut. Il serait cependant faux de prétendre que les adeptes du courant folklorique sont à l'abri des pressions financières exercées par l'industrie de la culture. D'une certaine manière, on

4. Loc. cit.

5. Il ne faudrait pas sous-estimer l'importance du bassin francophone hors Québec, qui représente un marché de plus d'un million de consommateurs.

6. L'expression est de Michel Wieviorka, La Démocratie à l'épreuve, nationalisme, populisme, ethnicité, Paris, La découverte, 1993. 
pourrait même affirmer que la chanson folklorique prête flanc à cette accusation, car, contrairement aux autres genres chansonniers dont les racines sont moins profondes, le « folklore » a, pour son malheur, ses puristes prompts à pointer l'infidèle du doigt : nous pensons ici encore au groupe Garolou. L'accusation est récurrente. "Ethnic business » signifie ici « trop innovateur », voire trop expressif, le sujet n'ayant pas su s'effacer devant l'œuvre !

Curieusement cependant, plus à gauche cette fois, l'accusation arborera le même vocable, mais pour des raisons différentes. L'œuvre n'est pas assez innovatrice ! C'est à ce point qu'il nous faut bien comprendre le sens de ce que Wieviorka appelle le triangle de l'ethnicité : chacun campé sur son pôle, celui des racines communautaires dans le premier cas et de l'innovation dans l'autre, les accusations se recoupent autour d'un troisième pôle que l'artiste fréquenterait de trop près: il s'agit ici du pôle de l'individu et de ses droits universels, susceptible celui-là de transformer l'individu en individualiste, le pragmatiste en opportuniste. Nous pensons ici en particulier à ces artistes acadiens et franco-canadiens qui doivent à l'occasion expliquer leurs absences au sein de leur communauté pour aller à Montréal ou à Paris, à la recherche de moyens de production équivalents à ceux d'autres artistes.

Le reproche qu'évoque ici l'ethnic business ne concerne pas tellement l'argent accumulé au moyen de l'art comme la somme des compromis admis pour exercer celui-ci. Or, il faut bien souligner que cette approche ne peut être considérée comme exclusive au «folklore ". Essentiellement de type « entrepreneurial », la stratégie correspond plutôt, pour une large part, au profil invoqué pour accoler au substantif « littérature » un attribut " populaire ». Pourtant, là encore, la fugacité des étiquettes rappelle davantage l'incessant procès typique des institutions littéraires qu'une frontière claire entre littérature consacrée et littérature populaire, c'est-à-dire, dans le cas qui nous occupe : la littérature orale étiquetée folklorique. Citons pour exemple le cas de l'écrivain Yves Thériault dont l'émergence en littérature est indissociable du procès institutionnel qui fit passer l'écrivain du statut d'écrivain populaire, vendeur de livres à quinze sous et au cycle de production relativement court, à un statut d'écrivain incontournable dans les manuels scolaires québécois. Le cas de Gilles Vigneault est aussi évocateur à ce sujet : à la publication dans les années soixante du premier recueil de chansons de celui qu'on appelait encore « la Bolduc de l'Université », Jean Éthier-Blais du Devoir posait la question : "Seriez-vous un poète Monsieur Vigneault?». Aujourd'hui, bien sûr, le doute ne serait pas sans risque. 


\section{Conclusion}

La mise sur pied d'un cours où littérature et folklore seraient mis en présence doit nécessairement impliquer que l'on prenne en compte les préjugés évoqués plus haut. Il s'agirait alors de montrer les différents visages d'un arrimage pas toujours reconnu entre identité et modernité, en somme de montrer que la matière dont il est question n'est pas morte, qu'elle est en fait inachevée et en devenir. Pour rendre compte de cette vie, il nous semble important de montrer et de démonter les divers mécanismes discursifs sources de dérives: " voir c'est montrer et montrer c'est changer ».

La citation de Sartre mérite pourtant ici d'être nuancée. Car il ne suffit pas de désirer le changement. Encore faut-il qu'au plan sociétal, celui-ci soit réalisable, voire souhaitable. La théorie des conflits de Weber force la réflexion ici autour du potentiel de politisation des conflits dans une communauté donnée. Chez Weber ${ }^{8}$, la perméabilité de la sphère politique à la sphère culturelle devient déterminante dans cette dynamique qui implique une croyance en un devoir faire (ou un devoir créer). De sorte que l'on pourrait penser qu'une accessibilité réduite à la sphère politique - comme c'est souvent le cas en milieu minoritaire - aurait pour effet de décourager la sphère culturelle de tout projet de réinvention de l'histoire de la communauté. Ce faisant, elle serait alors encline à se réfugier dans un passé dont la répétition ne serait que le seul avenir ; ce qui expliquerait alors la solitude d'un Zachary Richard engagé en Louisiane devant l'émergence d'un folklore dont les auteurs sont toujours vivants et pour lesquels Barry Ancelet a déjà créé une catégorie ou peut-être une tombe: «le faux vieux »!

7. Jean-Paul Sartre, Qu'est-ce que la littérature, Paris, Gallimard, 1985.

8. Max Webber, Économie et société (tome 1), Paris, Plon, 1971. 